\title{
Ventricular Remodeling in Rabbits with Sustained High-Fat Diet
}

\author{
Manuel Zarzoso, Sergey Mironov, Guadalupe Guerrero-Serna, B. Cicero Willis, Sandeep V.
} Pandit

\section{SUPPLEMENTAL METHODS}

\subsection{Animals and diet}

Experiments were performed using 15 New Zealand White rabbits (10-11 weeks old, $2.33 \pm 0.12 \mathrm{Kg}$ at the beginning of the study). Animal handling protocols conformed to United States National Institutes of Health Guidelines for the Care and Use of Laboratory Animals (National Institutes of Health publication $\mathrm{n}^{-}$85-23, revised 1996) and the European (D2003/65/CE and R2007/526/CE) Guidelines for the Care of Animals Used in Experimental and other Research Purposes, and were approved by the University Committee on Use and Care of Animals (UCUCA) of the University of Michigan, Ann Arbor.

The animals were housed under controlled conditions of temperature $\left(20 \pm 1.5^{\circ} \mathrm{C}\right)$ and humidity $(50 \pm 5 \%)$, with a 12 -h light cycle, and fed standard rabbit chow (Laboratory rabbit diet Purina 5326, LabDiet, MO, US). After a period of 2 weeks of acclimation the rabbits were randomly assigned to the control group $(C ; n=7)$, which was fed with the normal chow, or to the experimental group (HFD; $n=8$ ), which was fed with a high fat diet. The high-fat group was fed ad libitum with a high fat diet, which consisted of the standard rabbit chow with an additional $15 \%$ fat (10\% corn oil and 5\% lard, Research Diets Inc., NJ, US), as described previously [1]. Both groups were kept on their respective diets for 18 weeks.

Supplemental table 1 shows the composition and calories provided by the control chow and the high-fat chow. The standard rabbit chow used was Laboratory Rabbit Diet High Fiber \#5326 (LabDiet, St. Louis, MO, US) with the following ingredients: alfalfa meal, ground soybean hulls, wheat middlings, cane molasses, ground yellow corn, soybean meal, salt, calcium carbonate, dicalcium phosphate, animal fat preserved with BHA, vitamin A acetate, cyanocobalamin, choline chloride, calcium pantothenate, folic acid, nicotin acid, pyridoxine hydrochloride, DLmethionine, dl-alpha tocopheryl acetate, cholecalciferol, cobalt carbonate, riboflavin, copper sulfate, ferrous carbonate, magnesium oxide, manganous oxide, calcium iodate, zinc sulfate, zinc oxide and sodium selenite. Regarding the chemical composition, the control rabbit chow composition was: $14.5 \%$ protein, $2.2 \%$ fat, $21.1 \%$ fiber, $43.3 \%$ nitrogen-free extract and $18.9 \%$ other components. This represents a $23.1 \%$ of calories provided by proteins, $7.9 \%$ by fat and $69.1 \%$ by carbohydrates.

\subsection{Experimental protocol}

After the two weeks of acclimation, and before starting the diet, rabbits were placed in a rabbit restrainer and were anesthetized with intramuscular injection of Ketamine (KetaVed, 25 $\mathrm{mg} / \mathrm{kg}$ ) and Xylazine (Anased, $10 \mathrm{mg} / \mathrm{kg}$ ). Once the rabbits were sedated, and after waiting at least, a minimum of 10 minutes after the injection, blood glucose, electrocardiography and echocardiography studies were performed. The weight of the animals was monitored weekly. After 18 weeks, and 2-3 days before euthanizing the animals, the same procedure was followed 
and the blood glucose, electrocardiography and echocardiography measurements were performed, in addition to an intravenous glucose tolerance test and morphological measurements. When euthanasia was performed, and prior to the electrophysiological experiment in isolated rabbit heart, blood samples were collected.

\subsection{Morphological measurements}

Body measurements were taken by means of measuring tapes and a weighing scale before performing the glucose tolerance test. Body weight, body length, body mass index (BMI), abdominal circumference and abdominal circumference/body length ratio were determined.

\subsection{Intravenous glucose tolerance test}

For the evaluation of glucose metabolism, intravenous glucose tolerance test (IVGTT) was performed as previously described [2]. Rabbits were fasted overnight and the experiment started between 8 and 9 a.m. After anesthesia, a bolus of glucose $(600 \mathrm{mg} / \mathrm{kg})$ was administered i.v. through the marginal ear vein and blood samples were taken before and at different time points $(10,20,30,60,90,120$ and 240 minutes) after injection. Blood glucose was measured with a glucose meter (Precission Xtra, Abbot Diabetes Care, CA, US). The area under the curve (AUC) was calculated by multiplying the cumulative mean height of glucose (mg/dL) by the time (hours) [3].

\subsection{Echocardiographic study}

Transthoracic echocardiography was performed as in earlier studies [4] before and 18 weeks after starting the high fat diet, using Vevo 2100 ultrasound system (Visualsonics, ON) with a MS200 9-18 MHz Cardiovascular MicroScan transducer up to 30 micron resolution and frame rates in $2 \mathrm{D}$ up to 740 frames/second (for a $4 \times 4 \mathrm{~mm}$ field of view). Doppler, m-mode, tissue doppler and 2D protocols were used. The main parameters of analysis included ejection fraction, cardiac output, fractional shortening, left atrium (LA), left ventricle posterior wall (LVPW), interventricular septum (IVS) and left ventricle (LV), diameter, volume, mass and thickness. The parameters analyzed in each of the protocols are detailed in Supplemental table 2. Analyses were performed by an experienced echocardiographer (Kimber Converso-Baran) blinded to the study.

\subsection{Electrocardiographic study}

ECG recordings (of $500 \mathrm{~s}$ duration) were made by means of two standard bipolar leads connected to a P511 amplifier (Grass Technologies, RI, US) with a sampling rate of $1 \mathrm{kHz}$. The experiments were performed between 9 and 10 a.m., after anesthesia. P-P and R-R intervals, $\mathrm{P}$-wave duration and dispersion, QRS duration, $\mathrm{P}-\mathrm{R}$ interval and QT interval were analyzed with Clampfit ver. 10.2.0.12 (Molecular Devices, CA, US) and the mean of 25 consecutive beats was used in each animal for quantification. Corrected QT interval (QTc) was calculated according to the formula: QTc = QT-0.175(RR-300) [5]. QTc dispersion was assessed as the difference 
between the maximum and minimum QTc interval. Similarly, P wave dispersion was computed as the difference between the maximum and minimum P-wave duration.

Additionally, we quantified short-term heart rate variability (HRV) in 500 s recordings. Timedomain, geometrical and nonlinear standard HRV parameters were quantified using Kubios HRV software [6] (Supplemental table 3).

\subsection{Electrophysiological study}

Optical mapping was performed as described in previous studies [7,8,9]. Briefly, following sedation, rabbits were treated with heparin and anesthetized with sodium pentobarbital (50-100 $\mathrm{mg} / \mathrm{kg}$, administered intravenously via ear marginal vein). Upon reaching surgical-level anesthesia, hearts were quickly removed and immersed in cold $\left(4^{\circ} \mathrm{C}\right)$ cardioplegic solution for further preparation. The aorta was cannulated and connected to a Langendorff system to perfuse the heart with warmed, oxygenated Tyrode's solution ( $\mathrm{pH} 7.4$ ) containing (in mM) 130 $\mathrm{NaCl}, 5.4 \mathrm{KCl}, 1.8 \mathrm{CaCl}_{2}, 0.6 \mathrm{MgCl}_{2}, 1.4 \mathrm{NaH}_{2} \mathrm{PO}_{4}, 25 \mathrm{NaHCO}_{3}$, and 10 glucose. Oxygenation was carried out with a mixture of $95 \% \mathrm{O}_{2}$ and $5 \% \mathrm{CO}_{2}$. The heart was immersed in the same Tyrode's solution, in a custom-made plastic chamber, and the temperature of both the perfusate leaving the cannula and that in the superfusion chamber was maintained at $35.5 \pm 1.5^{\circ} \mathrm{C}$. The perfusion pressure was maintained between $60-70 \mathrm{~mm} \mathrm{Hg}$, by means of controlling the flow of the perfusate, at $\sim 30-40 \mathrm{ml} / \mathrm{min}$. Blebbistatin $(5-10 \mu \mathrm{M}$, Sigma) was added to the perfusate to uncouple electrical impulses from contraction, thus minimizing motion artifact. Blebbistatin has been shown to have minimal effect on rabbit ventricular electrophysiology [10]. A 1-2 ml bolus of the voltage-sensitive dye Di-4-ANEPPS (10 $\mu \mathrm{M}$, Sigma) was added to the perfusion line. Green light from a $532 \mathrm{~nm}, 1$ Watt laser was directed at the anterior surface of the heart and emitted light passed through a $650 \pm 50 \mathrm{~nm}$ filter before being captured by a high-resolution $80 \times 80$ pixel Little Joe CCD camera, at $1 \mathrm{kHz}$ sampling frequency. The typical duration of a recorded optical movie was 5 seconds.

Stimulation was performed by means of a silver wire bipolar electrode $(0.5 \mathrm{~mm}$ interelectrode distance, $0.25 \mathrm{~mm}$ electrode diameter) positioned on the right ventricle epicardium near the outflow tract. Electrical stimuli with an intensity 2 times the diastolic threshold were delivered by an A310 Accupulser stimulator (World Precision Instruments, FL, US) connected to a stimulus isolation unit (A385, World Precision Instruments, FL, US). Volume conducted ECGs (pseudo lead 1) were acquired at $1 \mathrm{KHz}$ using a P511 AC amplifier (Grass Technologies, RI, USA). The hearts were paced at 4, 4.4, 5, 5.7, 6.6, 8 and $10 \mathrm{~Hz}(250,225$, $200,175,150,125$, and $100 \mathrm{~ms}$ cycle length, respectively) under different extracellular potassium concentrations, in normo- and hyperkalemia (4, and $12 \mathrm{mM}\left[\mathrm{K}^{+}\right]_{0}$ respectively), and during perfusion with the beta adrenergic agonist isoproterenol (100 nM). Measurements of conduction velocity $(\mathrm{CV})$ of the impulse propagation in the heart show a classic biphasic response, when the extracellular $\mathrm{K}^{+}$levels are gradually increased; i.e. if extracellular $\mathrm{K}^{+}$is increased from 4 to 6-8 $\mathrm{mM}$, the CV actually increases, and then slows down as further increases in $\mathrm{K}^{+}$follow, i.e. > $10 \mathrm{mM}$ (see the classic experiments by Kagiyama et al. [17] in this regard; and also simulations that were able to reproduce this phenomenon [18]. Thus the choice of high $\mathrm{K}^{+}(12 \mathrm{mM})$ was dictated by the attempt to avoid the biphasic phase where CV increases, instead of slowing down. Ventricular fibrillation (VF) was triggered by pacing at 
increasing frequencies. Optical action potential duration (APD) at $80 \%$ of repolarization (APD80), conduction velocity (CV), dominant frequency (DF), regularity index (RI) and singularity points density (SPD) measurements were made. Analytical tools developed by members of our laboratory (Dr. Sergey Mironov: SCROLL) were used to calculate these parameters, as in previous studies [11,12].

\subsection{Parameters in plasma}

Postheparin plasma was collected from rabbits at week 18, 10 minutes after a bolus injection of heparin at a dose of $30 \mathrm{U} / \mathrm{Kg}$ body weight. Triglycerides (TG), total cholesterol (TC) and high-density-lipoprotein (HDL) cholesterol were determined by standard enzymatic procedures using reagent kits (Num. 3034658, 3313018 and 3034569, Roche Diagnostics, IN, US).

\subsection{Statistical analysis}

Values are reported as mean \pm standard deviation (SD) unless stated otherwise. A repeated measures analysis of variance model with Bonferroni test for multiple comparisons and unpaired t-test were used when appropriate (SPSS, version 17.0 for Windows). Differences were considered significant at a two-tailed alpha level of $p<0.05$. 
Supplemental Table 1. Composition and calories provided by the standard and high-fat chows.

\begin{tabular}{|c|l|c|c|c|c|}
\multicolumn{2}{c}{} & \multicolumn{1}{c}{ Control } & \multicolumn{2}{c|}{ High-fat } \\
\hline kcal & \multicolumn{1}{c|}{ Component } & $\mathbf{g}$ & Kcal & $\mathbf{g}$ & Kcal \\
\hline 2.03 & $\begin{array}{l}\text { LabDiet Laboratory Rabbit Diet High } \\
\text { Fiber \#5326 }\end{array}$ & 1000 & 2030 & 849.7 & 1724.9 \\
\hline 9 & Corn Oil & 0 & 0 & 100 & 900 \\
\hline 9 & Lard & 0 & 0 & 50 & 450 \\
\hline & Red dye & 0 & 0 & 0.3 & 0 \\
\hline & TOTAL & $\mathbf{1 0 0 0}$ & $\mathbf{2 0 3 0}$ & $\mathbf{1 0 0 0}$ & $\mathbf{3 0 7 5}$ \\
\hline & Kcal/g & $\mathbf{2 . 0 3}$ & & $\mathbf{3 . 0 7}$ \\
\hline
\end{tabular}


Supplemental Table 2. Description of the echocardiographic measurements.

\begin{tabular}{|c|c|}
\hline \multicolumn{2}{|l|}{ PARAMETER } \\
\hline \multicolumn{2}{|r|}{ Hemodynamics } \\
\hline Stroke volume & LV stroke volume, Endocardial vol; d - Endocardial vol; s \\
\hline Cardiac output & Cardiac output of the LV; endocardial SV * HR \\
\hline \multicolumn{2}{|r|}{ Systolic function } \\
\hline LV ejection fraction & 100 * ((LV Vol;d - LV Vol;s) / LV Vol;d) \\
\hline LV volume diastole & $\left((7.0 /(2.4+\right.$ LVID;d $)){ }^{*}$ LVID; $\mathrm{d}^{3}$ \\
\hline LV volume systole & Left ventricular internal diameter (systole) \\
\hline LV fractional shortening & 100 * ((LVID;d - LVID;s) / LVID;d) \\
\hline LV int. diameter diastole & Left ventricular internal diameter (diastole) \\
\hline LV int. diameter systole & $\left(\left(7.0 /(2.4+\right.\right.$ LVID;s) $){ }^{*}{\text { LVID } ; \mathrm{s}^{3}}^{3}$ \\
\hline \multicolumn{2}{|r|}{ Diastolic function } \\
\hline Mitral valve E vel. & Mitral valve E velocity \\
\hline Mitral valve A vel. & Mitral valve A velocity \\
\hline E' septal annulus vel. & Velocity at E' septal annulus \\
\hline Mitral valve E/A & Mitral valve $E$ to $A$ ratio \\
\hline Mitral valve E/E'septal anulus & Ratio of MV E velocity to E' velocity at septal annulus \\
\hline Isovolumic relax. time & Isovolumic relaxation time \\
\hline Isovolumic cont. time & Isovolumic contraction time \\
\hline \multicolumn{2}{|r|}{ Morphological parameters } \\
\hline IVS diastole & Inter ventricular septum thickness (diastole) \\
\hline IVS systole & Inter ventricular septum thickness (systole) \\
\hline LV posterior wall diastole & Left ventricular posterior wall thickness (diastole) \\
\hline LV posterior wall systole & Left ventricular posterior wall thickness (systole) \\
\hline LV mass & 1.053 * ((LVID;d + LVPW;d + IVS;d)3- LVID;d3) \\
\hline LA size & Left Atrium in end systole from parasternal long axis view \\
\hline LA volume & The volume of the LA at end systole (maximum size) \\
\hline
\end{tabular}


Supplemental Table 3. Description of the short-term HRV measurements.

\section{PARAMETER}

\section{DESCRIPTION}

Time domain analysis (statistical measurements)

\begin{tabular}{|l|l|}
\hline RR (ms) & Mean of the selected R-R interval series \\
\hline SDRR (ms) & Standard deviation of the R-R interval series \\
\hline RMSSD (ms) & $\begin{array}{l}\text { The root mean square of differences of successive R-R } \\
\text { intervals }\end{array}$ \\
\hline pNN50 (\%) & $\begin{array}{l}\text { The percentage value of consecutive R-R intervals that } \\
\text { differ more than 50 ms }\end{array}$ \\
\hline \multicolumn{2}{|c|}{ Time domain analysis (geometrical measurements) } \\
\hline HRVI (a.u.) & $\begin{array}{l}\text { HRV triangular index, the integral of the sample density } \\
\text { distribution of R-R intervals divided by the maximum of the } \\
\text { density distribution }\end{array}$ \\
\hline TIRR (ms) & $\begin{array}{l}\text { Triangular interpolation of the R-R interval histogram. } \\
\text { Baseline width of the minimum square difference triangular } \\
\text { interpolation of the maximum of the sample density } \\
\text { distribution of R-R intervals }\end{array}$ \\
\hline SD1 (ms) & $\begin{array}{l}\text { The standard deviation of Poincaré plot perpendicular to the } \\
\text { line of identity. It represents the instantaneous beat-to-beat } \\
\text { variability }\end{array}$ \\
\hline SD2 (ms) & $\begin{array}{l}\text { The standard deviation of Poincaré plot along the line of } \\
\text { identity. It represents the continuous long-term beat-to-beat } \\
\text { variability }\end{array}$ \\
\hline
\end{tabular}


(a)

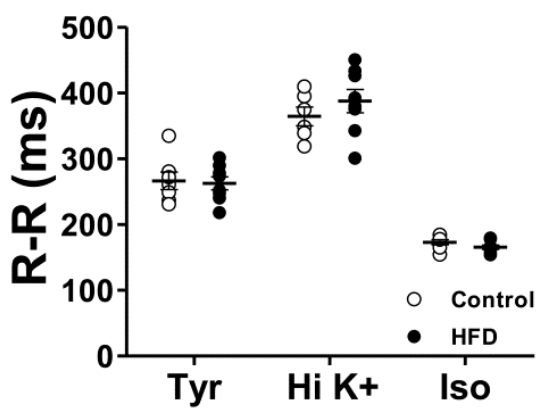

(d)

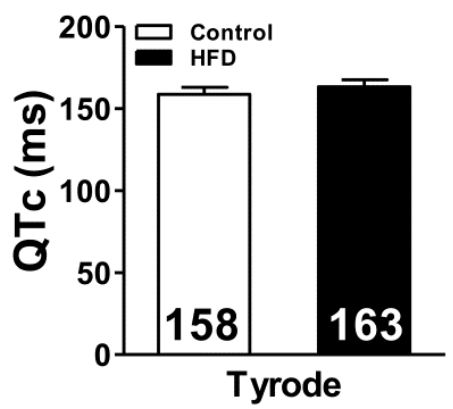

(b)

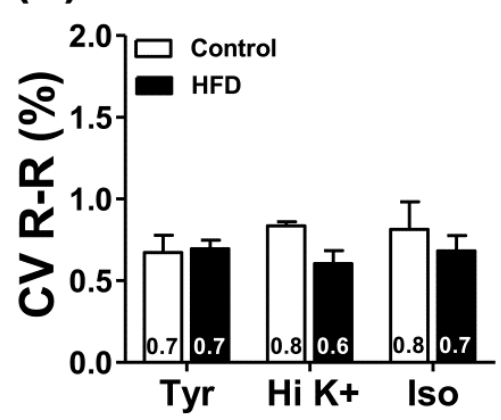

(e)

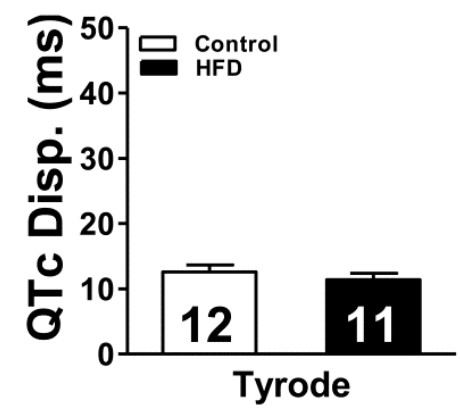

(c)

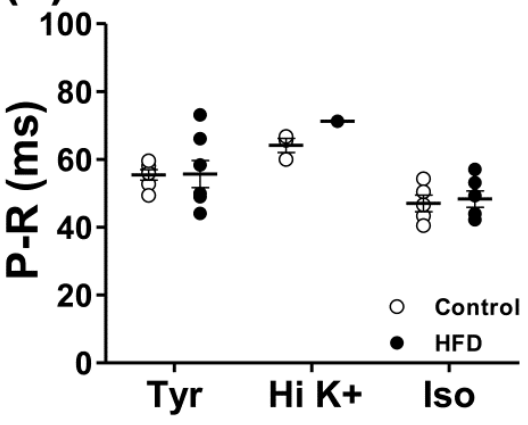

(f)

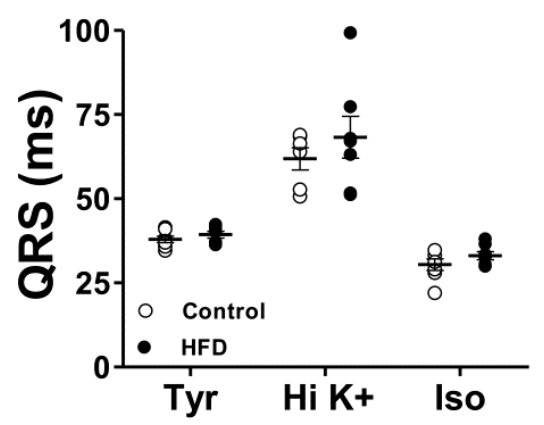

Supplemental Figure 1. Volume-conducted ECG parameters in optical mapping experiments. $R-R$ interval (a), Coefficient of variation of $R-R$ interval (b), P-R interval (c), corrected QT interval (d), QT interval dispersion (e) and QRS duration (f) in control $(n=5-7)$ and HFD $(n=5-8)$ groups. Error bars display the SEM. ${ }^{*} p<0.05$ vs control. Tyr $=$ during Tyrode perfusion; $\mathrm{Hi} \mathrm{K}_{+}=$during high potassium $(12 \mathrm{mM})$ perfusion; Iso = during isoproterenol (100 $\mathrm{nM}$ ) perfusion. 


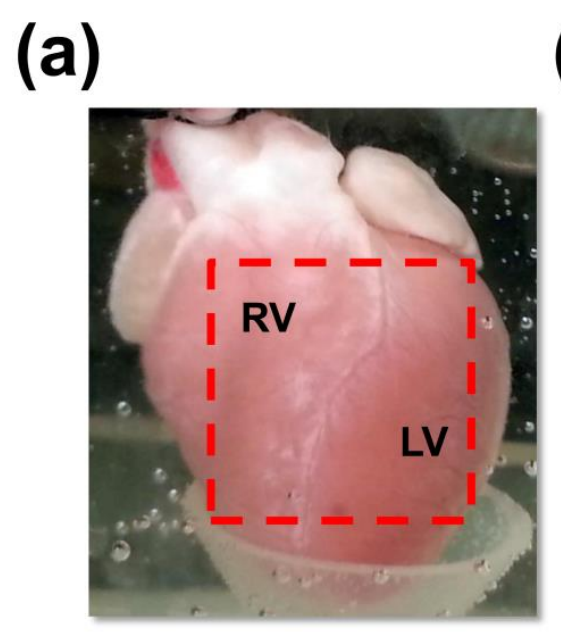

(c)

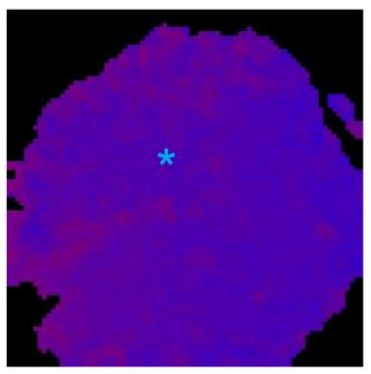

Tyrode

(b)

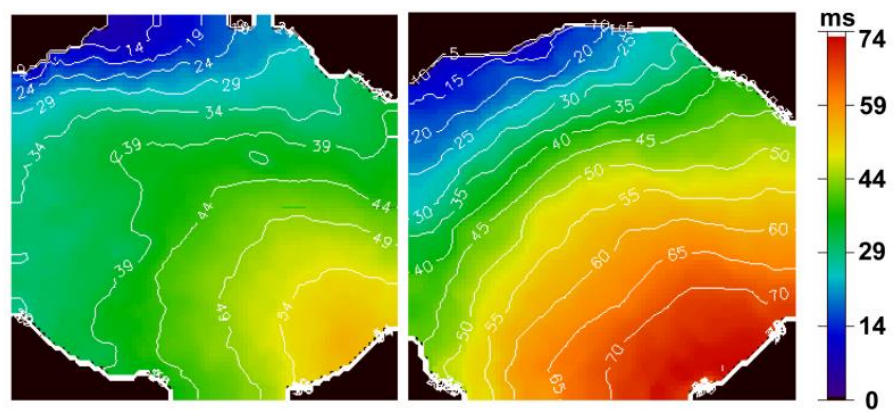

Tyrode

Hyperkalemia

(d)

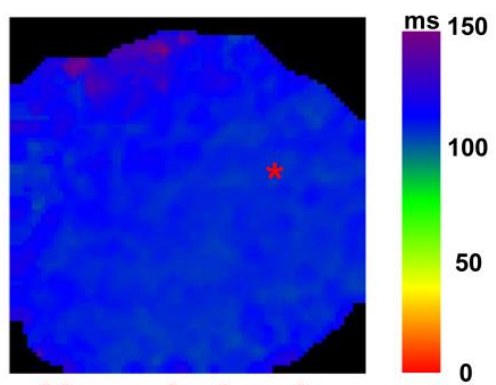

Hyperkalemia

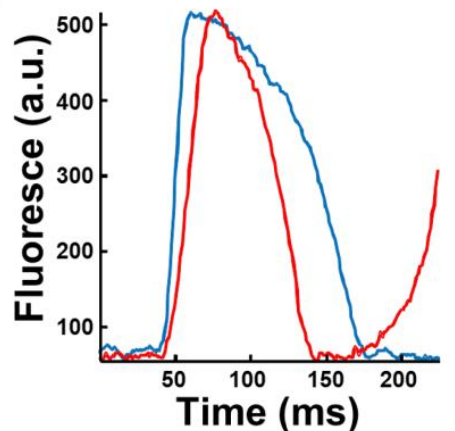

Supplemental Figure 2. Optical mapping data analysis and interpretation. Panel a shows an image of the isolated heart preparation (anterior view). The field of view of the camera is depicted by the red dashed line, and included the epicardial surface or the right (RV) and left ventricle (LV). Spatiotemporal filtering was applied to the movies using space filtering with $5 \times 5$ pixel kernel size, and a 11-frame temporal filter [12,13]. Spatial filtering was done with conical filters, and the temporal filtering was also carried out as weighted averages over the eleven frames with maximal weight on the central frame [12,13]. Subsequently, background fluorescence was subtracted. Activation maps are shown in panel b. Activation times (defined as the time at which each signal reached $50 \%$ of its upstroke amplitude, a value reached via interpolation from the recorded value above, and the value below the $50 \%$ amplitude) were determined for each pixel. Activation maps were constructed from activation times. Panel b shows typical activation maps during normal Tyrode perfusion and hyperkalemia, where we can observe the expected reduction in conduction velocity (isocrones $=5 \mathrm{~ms}$ ). Typical action potential duration maps at $80 \%$ of repolarization $\left(\mathrm{APD}_{80}\right)$ are shown in panel c. In panel d we superimposed examples of optical action potentials from single pixel recordings in the areas marked with an asterisk in panel c, in control (blue) and hyperkalemia (red), showing the expected reduction in APD during hyperkalemia. 
(a)

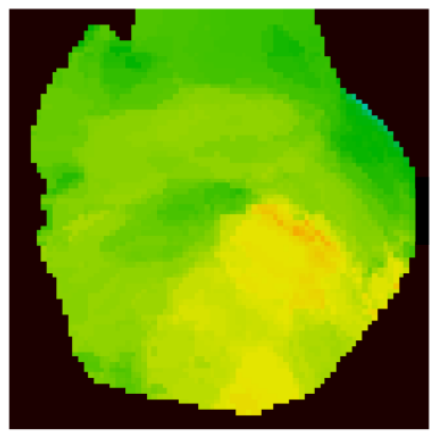

(c)

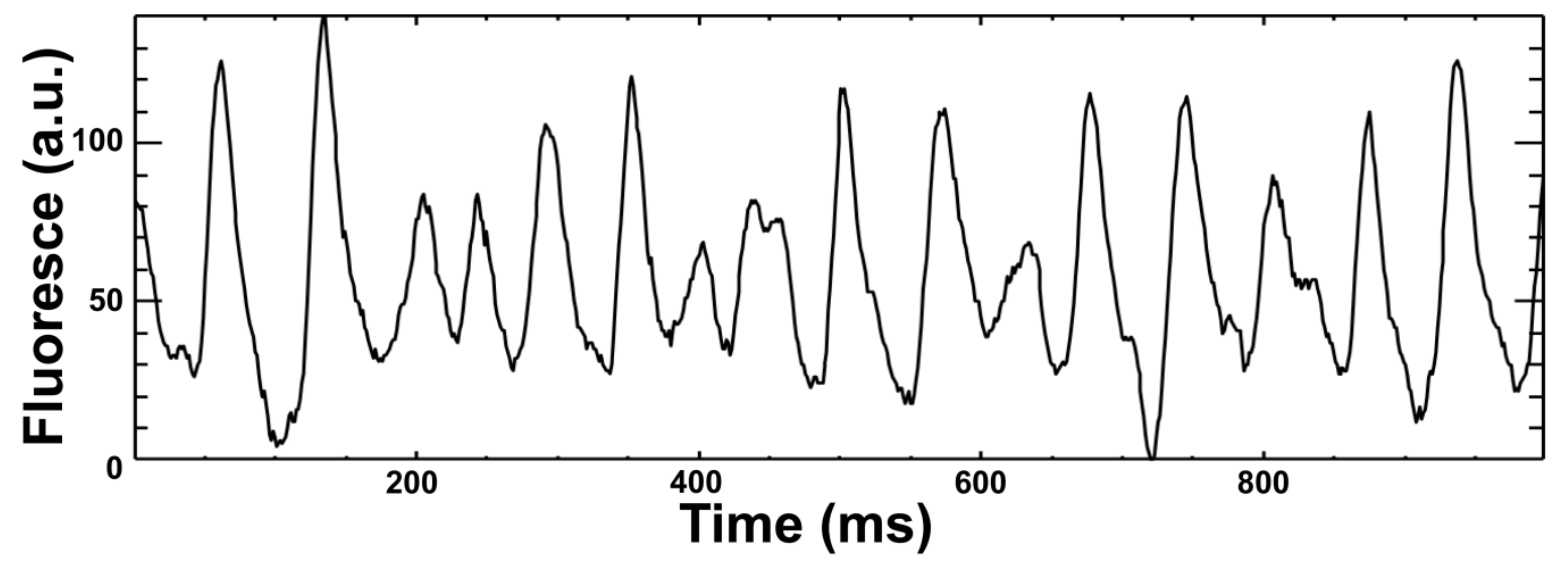

(b)

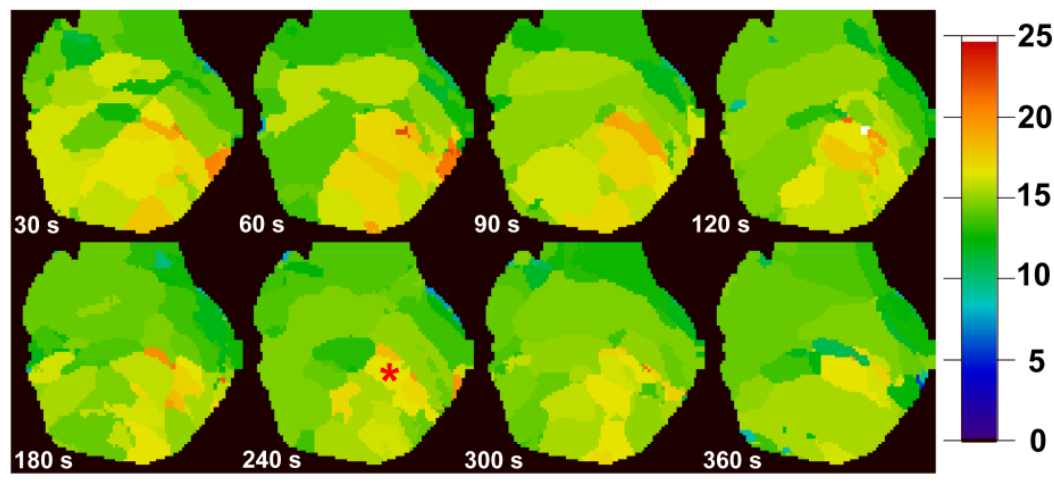

Supplemental Figure 3. Dominant frequency (DF) analysis of the isoproterenol-induced ventricular fibrillation (VF). For the time series of each pixel, the power spectrum density was estimated and the frequency with the maximum power was assigned to be the DF. For each episode, we obtained a global DF and pixel-by-pixel DFs. The latter was presented as a map that gave the spatial distribution of DFs in the field of view at each time point of the arrhythmic episode (b). The estimation of the power spectrum was obtained by the method of Welch [14]. Briefly, the time series was subdivided into two partially overlapping segments. The power spectrum of each segment (2.13 sec long) was obtained via fast Fourier transformation and then averaged and normalized to the total power. The outcome of this procedure was the removal of the inconsistent variability, enhanced weight of the peaks, and a frequency resolution of $0.47 \mathrm{~Hz}$. Thereafter, the $6 \mathrm{DF}$ maps of each movie in an experiment were superimposed to construct an averaged color-coded DF map for that experiment (a). Panel c displays the epicardial optical signal of the pixel highlighted in panel $\mathbf{b}$ at $240 \mathrm{~s}$ after VF initiation. 


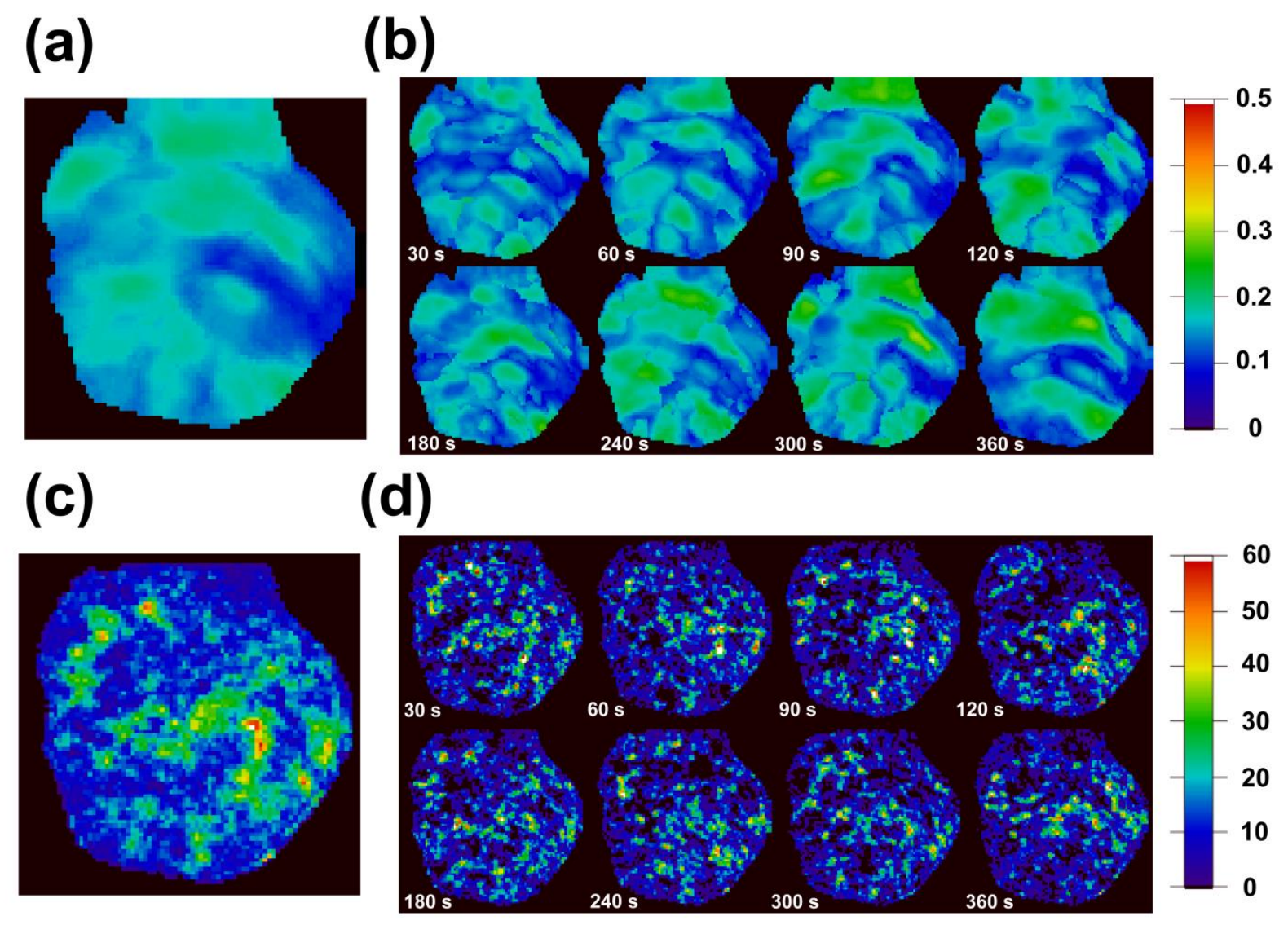

Supplemental Figure 4. Analysis of the spectral characteristics of the arrhythmia: regularity index and phase maps. For each signal, regularity index (RI) was defined as the ratio of the power at the DF to total power [11]. The power at the DF was calculated by summing the power values at the highest peak and its adjacent values (fixed band of $\sim 0.75 \mathrm{~Hz}$ ), and the total power was calculated as the sum over the range of 1 to $30 \mathrm{~Hz}$. Average maps of RI were constructed like the DF maps at each time point of the arrhythmic episode (b). Then, the $6 \mathrm{Rl}$ maps of each movie in an experiment were superimposed to construct an averaged color-coded DF map for that experiment (a). Phase movies were generated by applying the Hilbert transform to the fluorescent signals [15]. Singularity points (SPs) were defined as points where all phases converged [16], and were identified automatically in all VF movies. Average phase maps were constructed like the DF and RI maps at each time point of the arrhythmic episode (d), and 6 phase maps of each movie in an experiment were superimposed to construct an averaged color-coded SPs density map for that experiment (c). 


\section{REFERENCES}

1. Carroll JF, Dwyer TM, Grady AW, Reinhart GA, Montani JP, Cockrell K, et al. Hypertension, cardiac hypertrophy, and neurohumoral activity in a new animal model of obesity. Am $J$ Physiol 1996; 271: H373-378

2. Koike T, Liang J, Wang X, Ichikawa T, Shiomi M, Liu G et al. Overexpression of lipoprotein lipase in transgenic Watanabe heritable hyperlipidemic rabbits improves hyperlipidemia and obesity. J Biol Chem 2004; 279: 7521-7529

3. Liu E, Kitajima S, Higaki Y, Morimoto M, Sun H, Watanabe $T$ et al. High lipoprotein lipase activity increases insulin sensitivity in transgenic rabbits. Metabolism 2005; 54: 132-138

4. Herron TJ, Devaney E, Mundada L, Arden E, Day S, Guerrero-Serna G, Turner I, Westfall $\mathrm{M}$, Metzger JM. Ca2+-independent positive molecular inotropy for failing rabbit and human cardiac muscle by alpha-myosin motor gene transfer. FASEB J 2010; 24: 415-24

5. Carlsson L, Abrahamsson C, Andersson B, Duker G, Schiller-Linhardt G. Proarrhythmic effects of the class III agent almokalant: importance of infusion rate, QT dispersion, and early afterdepolarisations. Cardiovasc Res 1993; 27: 2186-2193

6. Niskanen JP, Tarvainen MP, Ranta-aho PO, Karjalainen PA (2004). Software for advanced HRV analysis. Comput Methods Programs Biomed; 76: 73-81

7. Pandit SV, Kaur K, Zlochiver S, Noujaim SF, Furspan P, Mironov S, et al. Left-to-right ventricular differences in I(KATP) underlie epicardial repolarization gradient during global ischemia. Heart Rhythm 2011; 8:1732-1739

8. Pandit SV, Zlochiver S, Filgueiras-Rama D, Mironov S, Yamazaki M, Ennis SR et al. Targeting atrioventricular differences in ion channel properties for terminating acute atrial fibrillation in pigs. Cardiovasc Res 2011; 89: 843-851

9. Zarzoso M, Rysevaite K, Milstein ML, Calvo CJ, Kean AC, Atienza F, et al. Nerves Projecting from the Intrinsic Cardiac Ganglia of the Pulmonary Veins Modulate Sinoatrial Node Pacemaker Function. Cardiovasc Res 2013 Apr 3 [Epub ahead of print]

10. Fedorov VV, Lozinsky IT, Sosunov EA, Anyukhovsky EP, Rosen MR, Balke CW, et al. Application of blebbistatin as an excitation-contraction uncoupler for electrophysiologic study of rat and rabbit hearts. Heart Rhythm 2007; 4:619-626

11. Kalifa J, Tanaka K, Zaitsev AV, Warren M, Vaidyanathan R, Auerbach D et al. Mechanisms of wave fractionation at boundaries of high-frequency excitation in the posterior left atrium of the isolated sheep heart during atrial fibrillation. Circulation 2006; 113: 626-633

12. Pandit SV, Warren M, Mironov S, Tolkacheva EG, Kalifa J, Berenfeld O et al. Mechanisms underlying the antifibrillatory action of hyperkalemia in Guinea pig hearts. Biophys $J$ 2010; 98: 2091-2101

13. Mironov SF, Vetter FJ, Pertsov AM. Fluorescence imaging of cardiac propagation: spectral properties and filtering of optical action potentials. Am J Physiol Heart Circ Physiol 2006; 291: H327-335

14. Welch PD: The use of fast Fourier transform for the estimation of power spectra: A method based on time averaging over short, modified periodograms. IEEE Trans Audio Electroacoust 1967; AU-15:70-73

15. Warren, M., P. Guha, O. Berenfeld, A. Zaitsev, J. Anumonwo , A. Dhamoon, S. Bagwe, S. Taffet and J. Jalife. Blockade of the inward rectifying potassium current terminates ventricular fibrillation in the guinea pig heart. J Cardiovasc Electrophysiol 2003; 14: 621-631 
16. Gray, R., A. Pertsov and J. Jalife. 1998. Spatial and temporal organization during cardiac fibrillation. Nature 1998; 392: 75-78

17. Kagiyama, Y., Hill, J.L., Gettes, L.S. 1982. Interaction of acidosis and increased extracellular potassium on action potential characteristics and conduction in guinea pig ventricular muscle. Circ Res 51, 614-623.

18. Shaw, R.M., Rudy, Y. 1997. Electrophysiologic effects of acute myocardial ischemia. A mechanistic investigation of action potential conduction and conduction failure. Circ Res 80, 124-138. 